Lentini et al.

\title{
A reassessment of DNA immunoprecipitation-based genomic profiling
}

Antonio Lentini ${ }^{1}$, Cathrine Lagerwall ${ }^{1}$, Svante Vikingsson ${ }^{2}$, Heidi K. Mjoseng ${ }^{3}$, Karolos Douvlataniotis $^{1}$, Hartmut Vogt ${ }^{1}$, Henrik Green ${ }^{2}$, Richard R. Meehan ${ }^{3}$, Mikael Benson ${ }^{1,4}$ \& Colm E. Nestor ${ }^{1,4}$

\begin{abstract}
Affiliations
${ }^{1}$ Department of Clinical and Experimental Medicine, Linköping University, Linköping, SE58183 Sweden.
\end{abstract}

${ }^{2}$ Department of Medical and Health Sciences, Linköping University, Linköping, SE58183 Sweden.

${ }^{3}$ MRC Human Genetics Unit at the Institute of Genetics and Molecular Medicine at the University of Edinburgh, Crewe Road, Edinburgh, EH4 2XU, UK

${ }^{4}$ These authors contributed equally to this work

Correspondence should be addressed to C.E.N. (colm.nestor@liu.se)

DNA immunoprecipitation sequencing (DIP-seq) is a common enrichment method for profiling DNA modifications in mammalian genomes. However, DIP-seq profiles often exhibit significant variation between independent studies of the same genome and from profiles obtained by alternative methods. Here we show that these differences are primarily due to intrinsic affinity of IgG for short unmodified DNA repeats. This pervasive experimental error accounts for 50 - $99 \%$ of regions identified as 'enriched' for DNA modifications in DIP-seq data. Correction of this error profoundly alters DNA modification profiles for numerous cell types, including mouse embryonic stem cells, and subsequently reveals novel associations between DNA modifications, chromatin modifications and biological processes. We propose new methodological guidelines that minimize the impact of these errors on future DIP-seq experiments and allow new insights to be made from the wealth of existing DIP-seq data.

Canonical DNA methylation in mammals involves the covalent attachment of a methyl group to cytosine to form 5-methylcytosine $(5 \mathrm{mC})$. The ability to establish and maintain DNA methylation patterns is essential for normal development in mammals, and aberrant DNA methylation is observed in numerous diseases, including all forms of cancer ${ }^{1}$. Comprehensive mapping of DNA methylation (5-methylcytosine, 5mC) in multiple species has been critical to establishing the relevance of methylation dynamics in gene regulation and chromatin organization $^{2-4}$. An effective method of generating genome-wide $5 \mathrm{mC}$ profiles couples antibody-based enrichment of methylated DNA fragments (MeDIP) with hybridization to DNA micro-arrays (MeDIP-chip) or high-throughput sequencing (MeDIP-seq) ${ }^{5}{ }^{6}$. Subsequent comparisons with nucleotide resolution bisulphite sequencing (BS) techniques produced broadly correlative DNA methylation data ${ }^{6,7}$. Unlike BS, the MeDIP-seq information is not contained in the read sequence itself, but in the enrichment or depletion of sequencing reads that map to specific regions of the genome ${ }^{7,8}$. Consequently, appropriate control samples are required, which typically correspond to the input genomic DNA before enrichment. The low cost of DIP-seq initially made it the method of choice in studies involving large numbers of samples. Subsequently the application of the DIP-seq technique has been extended to chart the genomic location of additional DNA modifications (5-hydroxymethylcytosine, 5hmC; 5- 
Lentini et al.

formylcytosine, 5fC; 5-carboxycytosine, 5caC; and 6-methyladenosine, 6mA) as their corresponding antibodies became available, elucidating their roles in the process of DNA methylation remodeling and gene regulation ${ }^{9-16}$. Interestingly, verification of DIP profiles by independent methods revealed several problems with the DIP-seq approach, including preferential enrichment of low $\mathrm{CG}$ content regions by the $5 \mathrm{mC}$ antibody ${ }^{17}$ and enrichment of highly modified regions by the $5 \mathrm{hmC}$ antibody ${ }^{18}$. In addition, we and others recently reported an enrichment of short tandem repeat (STR) sequences in hMeDIP assays ${ }^{19,} 20$. However, the origin of STR enrichment and the scale of its impact on DIP-seq data remained unknown.

Here we performed a systematic analysis of DIP-seq profiles generated with antibodies against multiple DNA modifications. We demonstrate that highly specific off-target binding to unmodified repetitive sequences is not limited to $5 \mathrm{hmC}$ antibodies but is an inherent technical error observed in all DIP-seq studies, irrespective of the target DNA modification, cell-type or organism. We reveal that between 50\% - 99\% of enriched regions in DIP-Seq data are false positives, the removal of which markedly affects our perception of methylation dynamics in mammals; altering the associations between DNA methylation and other genomic and epigenomic features. In addition to inherent errors in DIP-seq, we also observed that contamination of mammalian samples with $6 \mathrm{~mA}$ containing bacterial DNA may account for the conflicting findings relating to the location and abundance of $6 \mathrm{~mA}$ in mammalian genomes. Finally, we detail adjustments to existing DNA immunoprecipitation protocols and suggest novel computational approaches that will minimize the impact of these errors on future DIPseq experiments and allow new insights to be gained from the wealth of existing DIP-seq data.

\section{RESULTS}

\section{IgG has an intrinsic affinity for short tandem repeats in mammalian DNA}

To simplify comparison of DIP-seq results from separate studies we used a uniform computational pipeline (see online methods) to analyze published DIP-seq profiles of $5 \mathrm{mC}$, $5 \mathrm{hmC}, 5 \mathrm{fC}$ and $5 \mathrm{caC}$ (hereby referred to as ' 5 modC') in mouse embryonic stem cells (mESCs). The sensitivity and specificity of $5 \operatorname{modC}$ antibodies used in DIP-Seq is well established, with limited to undetectable cross-reactivity observed in dot-blot and ELISA assays 15, 19, 20 (Supplementary Fig. 1a, b). All analyzed datasets and their relationship to figures is outlined in Supplementary Table 1. This approach revealed a striking enrichment at short tandem repeats (STRs) in all 5modC DIP-seq datasets (Fig. 1a). Surprisingly, near identical enrichment patterns at STRs were observed in mESC DIP-seq generated with a non-specific mouse IgG antibody (Fig. 1a). The intersection of regions enriched for all 5 modC showed a 5.8 fold higher enrichment for IgG compared to non-enriched DNA (Input; $P=5.03 \times 10^{-5}$, T-test) whereas nonintersecting regions showed no difference (Supplementary Fig. 1c), suggesting that a proportion of the 5modC signal may be due to off-target binding of the antibodies. Indeed, genome-wide IgG enrichment could explain up to $55 \%$ of all 5 modC DIP-seq enriched loci in mESCs whereas Input explained a maximum of 3\% of enriched regions (Supplementary Fig. 1d). Significantly, overlapping $5 \mathrm{mC}, 5 \mathrm{hmC}$ and $\mathrm{IgG}$ regions were depleted of $\mathrm{CpG}$ dinucleotides compared to regions not overlapping IgG (Supplementary Fig. 1e). Although non-CpG methylation is known to occur in $\mathrm{mESCs}^{21,22}$, analysis of whole-genome bisulfite sequencing data ${ }^{21}$ confirmed that $\mathrm{CpHs}$ in these regions were primarily unmethylated (median 
Lentini et al.

methylated $\mathrm{CpHs}=0$ and 3 for $\mathrm{IgG}$ and $5 \mathrm{mC}$ regions, respectively) (Supplementary Fig. 1f) suggesting that all antibodies were binding highly specific regions of unmodified DNA during DIP experiments. We verified this by analyzing published DIP-seq data from Dnmt triple knockout (TKO) $\mathrm{mESCs}^{23}$ that lack DNA methyltransferase activity and revealed that the $5 \mathrm{hmC}$ antibody enriched similar regions to that of the IgG control (Fig. 1b and Supplementary Fig. 1g). We confirmed depletion of both $5 \mathrm{mC}$ and $5 \mathrm{hmC}$ in TKO compared to wild-type (WT) mESC DNA using mass spectrometry (Fig. 1c), verifying that the DIP-seq signals observed in TKO cells were independent of 5 modC status. $5 \mathrm{hmC}$-DIP followed by qPCR confirmed the enrichment of STRs in TKO mESCs lacking 5hmC (Fig. 1d, e). Significantly, 5hmC profiles generated from an independent, non-antibody based $5 \mathrm{hmC}$ enrichment technique ${ }^{24}(5 \mathrm{hmC}$ Seal) showed no enrichment over IgG regions (Fig. 1f) further implicating off-target binding of STRs by antibodies during DIP-seq

To identify specific IgG-bound sequences, we screened the raw sequencing reads from three IgG DIP-seq samples in mESCs for overrepresented sequences, which revealed that between 30 and $60 \%$ of all reads were significantly enriched for repetitive motifs compared to Input (Fig. 1g and Supplementary Table 2), including the previously reported CA-repeats ${ }^{19}$. This suggested that IgG antibodies may have an innate binding capacity for single stranded repetitive DNA sequences. Interestingly, one of the most enriched motifs in IgG sequences reads was the 6-base TTAGGG sequence, suggesting that IgG also binds telomeric DNA which is susceptible to the formation of non-duplex (four-stranded quadruplex) structures ${ }^{25}$. Not only were IgG-DIP enriched for repetitive motifs, but the enriched IgG motifs were highly similar between samples (average Pearson $r=0.72$ ) indicating that $\mathrm{IgG}$ binding is specific and reproducible (Supplementary Table 2). We observed similar repeat motifs in 5modC DIP-seq data from mESCs as well as a recently published study in mouse embryonic fibroblasts $(\mathrm{MEFs})^{26}(\mathrm{r} \mathrm{mESC}=0.75, \mathrm{r}$ MEF $=0.68$, Supplementary Table 2$)$, showing that off-target binding of STRs in DIP-seq is not limited to mESCs and is highly sequence dependent. Indeed, the only antibody based profiling technique that did not show enrichment over IgG enriched regions was cytosine-5-methylenesulfonate (CMS)-seq ${ }^{27}$ (Fig. 1f), which involves bisulfite conversion of all unmodified cytosines to thymine before immunoprecipitation with the antiCMS antibody. Consequently, all unmodified CA-repeats would be converted to TA-repeats. The lack of CA-repeat enrichment in CMS-Seq is thus strongly supportive of sequence-specific off-target binding of STRs by IgG antibodies. Taken together, our analyses indicates that native DNA immunoprecipitation libraries generated with multiple cytosine modification antibodies enriches for highly specific regions of unmodified repetitive DNA. This systematic error has resulted in extremely inaccurate attributions with respect to the genomic location of these modifications in mammals.

\section{IgG binding of DNA repeats explains the conflicting results of $6 \mathrm{~mA}$ profiling in vertebrates}

Next, we extended our analysis to a non-cytosine modification, 6-methyldeoxyadenosine $(6 \mathrm{~mA})$, that is abundant in many bacteria and recently characterized in invertebrates ${ }^{10,28-30}$. Its subsequent discovery in mammalian DNA has sparked an intense research effort to verify its location and characterize its function ${ }^{13,31}$. To determine if $6 \mathrm{~mA}$ DIP-seq studies have also been affected by off-target binding of STRs we compared profiles from primary mouse kidney cells ${ }^{13}$ to $\mathrm{mESC}$ IgG DIP-seq profiles. Again, $6 \mathrm{~mA}$ profiles showed a clear enrichment at STRs and were highly similar to the mESC IgG profile (Fig. 2a, b). Analysis of additional public 
Lentini et al.

datasets revealed that $6 \mathrm{~mA}$ DIP-seq data for Danio rerio ${ }^{14}$ and Xenopus laevis ${ }^{13}$ also showed similar off-target enrichment for the same STR motifs observed in 5modC DIP-seq, whereas the $6 \mathrm{~mA}$ rich genomes of C. elegans ${ }^{29}$ and E.coli $^{13}$ showed no enrichment for these motifs (Fig. 2c, d). Inter-species differences in STR enrichment reflected the frequency of STRs in the genome of each species (Fig. 2e). Surprisingly, data from a recent study of 6mA-DIP in $\mathrm{mESCs}^{31}$ displayed different enrichment profiles from both mouse kidney $6 \mathrm{~mA}$ - and mESC IgG-DIP-seq. Since the mESCs were cultured in vitro we wanted to exclude possible contamination of $6 \mathrm{~mA}$-rich bacteria such as Mycoplasma ${ }^{32,}{ }^{33}$. To test this we mapped sequencing data to a combined genome index of M.musculus, Mycosplasma sp. and E.coli as a control (see Online Methods). This revealed that up to $15 \%$ of DIP-seq reads from the $6 \mathrm{~mA}$ mESC study ${ }^{31}$ mapped to Mycoplasma whereas 24 samples from four other studies ${ }^{13,15,23,26}$ mapped exclusively to M.musculus (Fig. 2f). Contamination of these samples may explain the earlier detection of $6 \mathrm{~mA}$ in mammalian samples by mass spectrometry ${ }^{31}$ and the subsequent failure of more recent attempts to detect $6 \mathrm{~mA}$ in mammalian DNA using ultrasensitive UHPLC-MS ${ }^{28,34}$.

\section{Normalizing for off-target STR binding sharpens our view of epigenetic organization in mammals}

Whereas the use of an appropriate IgG control sample would normalize for the effect of enrichment of unmodified STRs during DIP, the vast majority (> 96\%) of published DIP-seq studies do not use an IgG control. To determine how STR binding in DIP-seq has affected our understanding of DNA methylation in mammals, we reanalyzed data from five independent studies of 5 modC marks in $\operatorname{mESCs}^{15,19,23,35,36}$. First, we estimated the fraction of falsely enriched regions when using Input as a control, finding that up to $99 \%$ of enriched $5 \mathrm{fC}$ and $5 \mathrm{caC}$, and approximately half of all $5 \mathrm{hmC}$ and $5 \mathrm{mC}$ regions could be considered false positives (Fig. 3a). In contrast, the mean percentage of falsely enriched regions was only $5.6 \%$ for all 5 modC marks when using IgG as a control (Fig. 3a). These results suggested that the 5 modC landscape in mammalian genomes has been greatly overestimated by DIP-seq (Supplementary Fig. 2a, b). Indeed, correcting for IgG not only reduced the number of enriched regions but also greatly increased the overlap with CMS and Seal profiling techniques (Supplementary Fig. 2c). Not surprisingly, the proportion of enriched repeat types was markedly altered when using Input or IgG controls in DIP-Seq, with STRs showing the greatest changes in enrichment (Supplementary Fig. 2d). Interestingly, in addition to CA/GT repeats, AG/CT repeats were also highly enriched in Input controlled studies (Supplementary Fig. 2e). Off-target binding of AG rich motifs may explain the reported association of $6 \mathrm{~mA}$ with specific AG-rich repeats $\left(\mathrm{AGGG}_{\mathrm{N}}\right)^{13}$ which was highly similar to those observed for all 5modC-DIP and IgG-DIP in mESCs (Supplementary Table 2).

Having shown that correction for STR-binding markedly altered the profiles of all 5 modC in mESCs, we next investigated if these altered profiles impacted on their predicted roles in DNA de-methylation and gene regulation. Globally, $49 \%$ of $5 \mathrm{mC}$ - co-located with $5 \mathrm{hmC}$ enriched regions when using Input, whereas only $17 \%$ were coincident for both $5 \mathrm{hmC}$ and $5 \mathrm{mC}$ when using IgG (Fig. 3b). This suggested a more restricted role for $5 \mathrm{hmC}$ mediated DNA demethylation in the reprogramming of the mESC epigenome, an assertion supported by the markedly improved association between $5 \mathrm{hmC}$ and TET protein occupancy in the mESC genome upon normalization to IgG (Fig. 3c). Significantly, removal of signals caused by STRbinding by normalization to $\mathrm{IgG}$ also altered the association of $5 \mathrm{hmC}$ with biological pathways from non-significant associations with unrelated processes including 'cilia formation', 'smell perception' and 'phosphorus metabolism' to highly significant associations with processes 
Lentini et al.

related to mammalian development and cell differentiation (Fig. 3d, upper panels). Significantly, the $5 \mathrm{hmC}$-associated biological processes identified after correction for STRbinding were highly similar to those obtained with 5hmC-Seal and CMS-Seq, which do not enrich for unmodified repeats (Fig. 3d, lower panels). An improved association with developmental and differentiation related processes was also observed when the same correction was applied to mouse embryonic fibroblast cells (Supplementary Fig. 2f).

Finally, histone ChIP-seq data in mESCs from ENCODE ${ }^{37}$ showed no difference in enrichment between IgG DIP-seq enriched regions and randomly sampled regions (Fig. 3e) suggesting that repeats found in intact chromatin structures are not bound by $\mathrm{IgG}$, possibly due to their inability to form secondary structures. Again, using an IgG control significantly increased the association of $5 \mathrm{hmC}$ with permissive histone marks in $\mathrm{mESCs}^{37}$ whereas the association with heterochromatin (H3K9me3) decreased (Fig. 3f). For 5mC, the association with histone marks was also significantly increased, accentuating co-localization with $\mathrm{H} 3 \mathrm{~K} 9 \mathrm{me} 3$ as well as H3K36me3 which together with $5 \mathrm{mC}$ is involved in mRNA splicing ${ }^{38}$ (Fig. 3f). Taken together, these findings further highlight the profound effect off-target STR binding has had on our understanding of the interrelationship between 5 modC with mammalian chromatin signatures but also demonstrates the wealth of novel discoveries to be made by re-analysis of the vast body of published DIP-seq data.

\section{DISCUSSION}

Our reanalysis of published DIP-seq data revealed that all commonly used DIP-seq antibodies bind unmodified short tandem repeat (STR) sequences. By analyzing DIP-seq data from mouse embryonic stem cells (mESCs) lacking both $5 \mathrm{mC}$ and $5 \mathrm{hmC}$ we confirmed that STR binding was modification independent; as non-specific IgG antibody generated profiles highly similar to that of $5 \mathrm{mC}$ and $5 \mathrm{hmC}$ antibodies. Consequently, only studies that have normalized DNA modification enrichment to an IgG control have corrected for off-target binding ${ }^{15,23}(\text { Fig. 4) })^{15}$, 23. Unfortunately, greater than $96 \%$ of published DIP-seq studies do not include an IgG control. We showed that between 50 to $99 \%$ of enriched regions are due to off-target STR binding in 5 modC DIP studies, with studies of low abundance modifications (i.e. $5 \mathrm{caC} \& 5 \mathrm{fC}$ ) having the highest false positive rates. Although our findings require the field to re-visit published DIPseq data, they also provide an exciting opportunity to rapidly further our understanding of the dynamics of DNA methylation in mammalian biology by re-assessing the wealth of published DIP-seq data deposited in public databanks. Controlling for off-target IgG binding increased the signal to noise ratio in DIP-seq assays >3-fold, allowing identification of more subtle alterations in modification levels. This also results in a significantly smaller and more distinct epigenomic landscape in mammalian cells, evidenced by a significantly reduced overlap between $5 \mathrm{mC}$ and $5 \mathrm{hmC}$ marked loci and a stronger association between $5 \mathrm{modC}$ and a variety of chromatin marks (Fig. 3).

The prevalence of non-enriched Input DNA as a control in DIP-Seq studies stems from its use in ChIP-seq; Input chromatin helps to control for the different shearing dynamics of closed and open chromatin and for differences in the amplification efficiency of DNA fragments with different base compositions ${ }^{39}$. The preference for Input controls was also fueled by the requirement of a uniform background signal in early peak-calling algorithms ${ }^{40}$. However, Input does not control for non-specific antibody binding. Thus, we strongly suggest that all future DIP-Seq studies perform both an Input and IgG control that will allow for normalization of the 
Lentini et al.

effects of sequencing bias and antibody cross-reactivity ((IP-IgG)/Input) ${ }^{15,23}$. Indeed, future profiling studies of DNA modifications may be advised to use non-antibody based mapping techniques where possible ${ }^{41}$. Bisulfite sequencing (BS) of $5 \mathrm{mC}$ and oxidative BS or TAB-Seq of $5 \mathrm{hmC}$ offer quantitative, base-resolution alternatives to (h)MeDIP-seq, but remain prohibitively expensive ${ }^{42,43}$. The click chemistry based assays, $5 \mathrm{hmC}-\mathrm{Seal}$ and $5 \mathrm{fC}-\mathrm{Seal}$, are low-cost enrichment based techniques that do not exhibit STR enrichment bias, but may be less sensitive than their antibody-based counterparts ${ }^{16,20,24}$.

Whereas normalization of DIP-Seq data to an IgG-Seq control represents the optimal approach to generating accurate DIP-Seq profiles, IgG controls are lacking for the majority of published studies. Computational correction of published DIP-Seq data by filtering out sequencing reads containing IgG associated STR motifs is relatively straightforward but is not advised. First, as DNA modifications $(5 \mathrm{mC}, 5 \mathrm{hmC}, 5 \mathrm{fC})$ do occur at non-CpG dinucleotides in some cell types, complete removal of IgG-STR sequences may result in a loss of biologically significant information $^{21,} 26$ (Supplementary Fig. 2c, d). Second, as genomic STR composition differs markedly between species, the set of STRs bound by IgG and the extent of their enrichment is likely to vary in DIP-seq of DNA from different organisms (Fig. 2e). Third, as the effect of off-target STR binding increases with decreasing abundance of the target epitope (Fig. 3a), $a$ priori knowledge of global modification levels in each genome would be required to prevent over-correction of the data. Finally, other experimental variables such as antibody source and sensitivity, DNA denaturation conditions, stringency of washing may also effect the degree of STR-binding observed. Consequently, optimal reanalysis of published DIP-seq data requires the generation of additional IgG-Seq data for each cell type under investigation.

Unexpectedly, we also revealed the potential for contaminating bacterial DNA to confound the results of DIP-Seq studies of trace DNA modifications. The risk of such contaminants has been previously raised with regards to $6 \mathrm{~mA}^{10}$, which is vanishingly rare in mammals, but highly abundant in many bacterial species that commonly infect mammalian cell cultures, such as Mycoplasma and E.coli. Fortunately, even minor bacterial contamination of mammalian DNA samples can be identified by comparison of next generation sequencing reads with the genomic sequence of suspected contaminants. Using this approach, we found that up to $15 \%$ of reads in published samples of 6mA-DIP-seq in mammals mapped to the Mycoplasma genome ${ }^{31}$. Taken together with the results of a recent study that was unable to detect $6 \mathrm{~mA}$ in mammalian cells using mass spectrometry and our results showing clear enrichment for STRs using the $6 \mathrm{~mA}$ antibody, a re-evaluation of the extent and origin of $6 \mathrm{~mA}$ in mammalian studies is advisable ${ }^{34}$. As many bacteria and viruses contain abundant amounts of modified bases including $5 \mathrm{mC}$, $5 \mathrm{hmC}$ and $6 \mathrm{~mA}$, controlling for contaminating DNA in all DIP-seq assays requires more rigorous application, particularly when the genomic content of target modifications in mammals becomes increasingly rare.

How specific ssDNA molecules become bound to IgG during DNA immunoprecipitation is unclear? It would seem unlikely that the antigen-binding site of antibodies raised against different epitopes $(5 \mathrm{mC}, 5 \mathrm{hmC}, 5 \mathrm{fC}, 5 \mathrm{caC}$ and $6 \mathrm{~mA})$ would all exhibit affinity for the same ssDNA molecules. Alternatively, ssDNA molecules may bind directly to the conserved Fc region of IgG antibodies. Indeed, both ssRNA and ssDNA molecules ('aptamers') capable of specifically binding the Fc-region of mouse and rabbit IgG have been reported ${ }^{44}$. Aptamer binding to the $\mathrm{Fc}$ regions is highly secondary structure dependent ${ }^{44}$, which may explain enrichment for specific repetitive sequences that have a high probability of forming secondary 
Lentini et al.

structures during the DNA denaturation step of DIP. A role for secondary structure in STRenrichment is further supported by a recent study employing immunoprecipitation of drosophila RNA with a $5 \mathrm{hmC}$ antibody (hMeRIP-seq) that found $64 \%$ of enriched regions were coincident with AG-rich repetitive sequences ${ }^{45}$. It is tempting to speculate on a function of ssDNA-antibody binding in vivo as mechanism to recognize highly structured, single-stranded viral DNA.

Whereas our discovery of unmodified STR binding by IgG has revealed a serious flaw in DIPseq to date, it will allow the field to minimize the impact of these errors on future DIP based assays and accelerate the discovery of novel findings from the multitude of existing DIP-seq data.

\section{METHODS}

Methods and associated references are available in the online version of the paper.

\section{ACKNOWLEDGEMENTS}

This work was supported by the Swedish Research Council, Ake Wibergs Fund and the LiUCancer Network (C.E.N.). R.R.M. and H.J.M. was supported by the Medical Research Council, UK (MC_PC_U127574433).

\section{AUTHOR CONTRIBUTIONS}

C.L., S.V., K.D., and H.K.M. performed experiments, A.L., C.E.N. and S.V. analyzed data, A.L. R.R.M. and C.E.N. wrote the manuscript and H.V., H.G., R.R.M., M.B. and C.E.N. supervised the work.

\section{COMPETING FINANCIAL INTERESTS}

The authors declare no conflicts of interest.

\section{REFERENCES}

1. Goll, M.G. \& Bestor, T.H. Eukaryotic cytosine methyltransferases. Annual review of biochemistry 74, 481-514 (2005).

2. Bogdanovic, O. et al. Active DNA demethylation at enhancers during the vertebrate phylotypic period. Nat Genet 48, 417-426 (2016).

3. Feinberg, A.P. \& Tycko, B. The history of cancer epigenetics. Nat Rev Cancer 4, 143153 (2004).

4. Illingworth, R.S. et al. Orphan $\mathrm{CpG}$ islands identify numerous conserved promoters in the mammalian genome. PLoS Genet 6, e1001134 (2010).

5. Weber, M. et al. Chromosome-wide and promoter-specific analyses identify sites of differential DNA methylation in normal and transformed human cells. Nat Genet 37, 853-862 (2005).

6. Harris, R.A. et al. Comparison of sequencing-based methods to profile DNA methylation and identification of monoallelic epigenetic modifications. Nat Biotechnol 28, 1097-1105 (2010).

7. Bock, C. et al. Quantitative comparison of genome-wide DNA methylation mapping technologies. Nat Biotechnol 28, 1106-1114 (2010).

8. Bock, C. Analysing and interpreting DNA methylation data. Nat Rev Genet 13, 705719 (2012). 
Lentini et al.

9. Shen, L., Song, C.X., He, C. \& Zhang, Y. Mechanism and function of oxidative reversal of DNA and RNA methylation. Annual review of biochemistry 83, 585-614 (2014).

10. Traube, F.R. \& Carell, T. The chemistries and consequences of DNA and RNA methylation and demethylation. RNA Biol, 1-9 (2017).

11. Koh, K.P. \& Rao, A. DNA methylation and methylcytosine oxidation in cell fate decisions. Curr Opin Cell Biol 25, 152-161 (2013).

12. Wu, X. \& Zhang, Y. TET-mediated active DNA demethylation: mechanism, function and beyond. Nature reviews. Genetics 18, 517-534 (2017).

13. Koziol, M.J. et al. Identification of methylated deoxyadenosines in vertebrates reveals diversity in DNA modifications. Nature structural \& molecular biology 23, 24-30 (2016).

14. Liu, J. et al. Abundant DNA 6mA methylation during early embryogenesis of zebrafish and pig. Nat Commun 7, 13052 (2016).

15. Shen, L. et al. Genome-wide analysis reveals TET- and TDG-dependent 5methylcytosine oxidation dynamics. Cell 153, 692-706 (2013).

16. Song, C.X. et al. Genome-wide profiling of 5-formylcytosine reveals its roles in epigenetic priming. Cell 153, 678-691 (2013).

17. Nair, S.S. et al. Comparison of methyl-DNA immunoprecipitation (MeDIP) and methyl-CpG binding domain (MBD) protein capture for genome-wide DNA methylation analysis reveal $\mathrm{CpG}$ sequence coverage bias. Epigenetics 6, 34-44 (2011).

18. Ko, M. et al. Impaired hydroxylation of 5-methylcytosine in myeloid cancers with mutant TET2. Nature 468, 839-843 (2010).

19. Matarese, F., Carrillo-de Santa Pau, E. \& Stunnenberg, H.G. 5Hydroxymethylcytosine: a new kid on the epigenetic block? Mol Syst Biol 7, 562 (2011).

20. Thomson, J.P. et al. Comparative analysis of affinity-based 5-hydroxymethylation enrichment techniques. Nucleic Acids Res 41, e206 (2013).

21. Habibi, E. et al. Whole-genome bisulfite sequencing of two distinct interconvertible DNA methylomes of mouse embryonic stem cells. Cell Stem Cell 13, 360-369 (2013).

22. Ramsahoye, B.H. et al. Non-CpG methylation is prevalent in embryonic stem cells and may be mediated by DNA methyltransferase 3a. Proc Natl Acad Sci U S A 97, 5237-5242 (2000).

23. Williams, K. et al. TET1 and hydroxymethylcytosine in transcription and DNA methylation fidelity. Nature 473, 343-348 (2011).

24. Song, C.X. et al. Selective chemical labeling reveals the genome-wide distribution of 5-hydroxymethylcytosine. Nat Biotechnol 29, 68-72 (2011).

25. Parkinson, G.N., Lee, M.P. \& Neidle, S. Crystal structure of parallel quadruplexes from human telomeric DNA. Nature 417, 876-880 (2002).

26. Papin, C. et al. Combinatorial DNA methylation codes at repetitive elements. Genome Res 27, 934-946 (2017).

27. Pastor, W.A. et al. Genome-wide mapping of 5-hydroxymethylcytosine in embryonic stem cells. Nature 473, 394-397 (2011).

28. Fu, Y. et al. N6-methyldeoxyadenosine marks active transcription start sites in Chlamydomonas. Cell 161, 879-892 (2015).

29. Greer, E.L. et al. DNA Methylation on N6-Adenine in C. elegans. Cell 161, 868-878 (2015).

30. Zhang, G. et al. N6-methyladenine DNA modification in Drosophila. Cell 161, 893906 (2015). 
Lentini et al.

31. Wu, T.P. et al. DNA methylation on N(6)-adenine in mammalian embryonic stem cells. Nature 532, 329-333 (2016).

32. Razin, A. \& Razin, S. Methylated bases in mycoplasmal DNA. Nucleic acids research 8, 1383-1390 (1980).

33. Lluch-Senar, M. et al. Comprehensive methylome characterization of Mycoplasma genitalium and Mycoplasma pneumoniae at single-base resolution. PLoS genetics $\mathbf{9}$, e1003191 (2013).

34. Schiffers, S. et al. Quantitative LC-MS Provides No Evidence for $\mathrm{m} 6 \mathrm{dA}$ or $\mathrm{m} 4 \mathrm{dC}$ in the Genome of Mouse Embryonic Stem Cells and Tissues. Angew Chem Int Ed Engl (2017).

35. Ficz, G. et al. Dynamic regulation of 5-hydroxymethylcytosine in mouse ES cells and during differentiation. Nature 473, 398-402 (2011).

36. $\mathrm{Xu}, \mathrm{Y}$. et al. Genome-wide regulation of $5 \mathrm{hmC}, 5 \mathrm{mC}$, and gene expression by Tet1 hydroxylase in mouse embryonic stem cells. Mol Cell 42, 451-464 (2011).

37. Yue, F. et al. A comparative encyclopedia of DNA elements in the mouse genome. Nature 515, 355-364 (2014).

38. Brown, S.J., Stoilov, P. \& Xing, Y. Chromatin and epigenetic regulation of premRNA processing. Human molecular genetics 21, R90-96 (2012).

39. Kidder, B.L., Hu, G. \& Zhao, K. ChIP-Seq: technical considerations for obtaining high-quality data. Nature immunology 12, 918-922 (2011).

40. Laajala, T.D. et al. A practical comparison of methods for detecting transcription factor binding sites in ChIP-seq experiments. BMC genomics 10, 618 (2009).

41. Nestor, C.E., Reddington, J.P., Benson, M. \& Meehan, R.R. Investigating 5hydroxymethylcytosine $(5 \mathrm{hmC})$ : the state of the art. Methods in molecular biology 1094, 243-258 (2014).

42. Booth, M.J. et al. Quantitative sequencing of 5-methylcytosine and 5hydroxymethylcytosine at single-base resolution. Science 336, 934-937 (2012).

43. Yu, M. et al. Base-resolution analysis of 5-hydroxymethylcytosine in the mammalian genome. Cell 149, 1368-1380 (2012).

44. Nezlin, R. Aptamers in immunological research. Immunology letters 162, 252-255 (2014).

45. Delatte, B. et al. RNA biochemistry. Transcriptome-wide distribution and function of RNA hydroxymethylcytosine. Science 351, 282-285 (2016).

\section{FIGURE LEGENDS}

Figure 1. Characterization of off-target antibody binding in DIP-seq. (a) Signal track in mESCs showing similar enrichment between 5modC and IgG DIP-seq samples over repetitive regions. WGBS, whole-genome bisulfite sequencing; STRs, short tandem repeats. (b) Signal track of 5hmC and IgG DIP-seq in hypomethylated Dnmt triple knockout (TKO) or methylated wildtype (WT) mESCs over 5hmC- (left) or IgG enriched regions (right). (c) Mass spectrometry 
Lentini et al.

quantification of $5 \mathrm{mC}$ and $5 \mathrm{hmC}$ in TKO and WT mECSs showing their depletion in TKO. Bars represent mean \pm s.d of 3 biological replicates. $* * *$ P-value $<0.001$, T-test. (d) DIP-qPCR measurement of $5 \mathrm{hmC}$ levels in Dnmt wild-type (WT) mESCs over positive and negative control regions. Bars represent mean \pm s.d of 3 biological replicates. (e) DIP-qPCR measurement using a $5 \mathrm{hmC}$ antibody in Dnmt triple knockout (TKO) mESCs showing offtarget enrichment over repetitive regions. Bars represent mean \pm s.d of 3 biological replicates, correlation calculated using Spearman correlation. STRs, short tandem repeats. (f) $5 \mathrm{hmC}$ enrichment in mESCs with different profiling techniques over $5 \mathrm{hmC}$ - (left) or IgG enriched regions (right) showing specific DIP-seq enrichment over IgG regions. (g) Motif enrichment for raw IgG reads compared to Input showing enrichment of repetitive sequences of short tandem repeat (STR) and satellite (SAT) classes of repeats.

Figure 2. Characterization of similarities between $6 \mathrm{~mA}$ and IgG DIP-seq in different species. (a) Signal track for Input and 6mA DIP-seq in mouse kidney cells and IgG DIP-seq in mESCs. (b) 6mA DIP-seq and Input enrichment over IgG enriched DIP-seq regions. Bars represent mean \pm s.d of 3 biological replicates. P-values calculated using T-test. STRs, short tandem repeats (c) Motif enrichment for raw 6mA or IgG DIP-seq reads compared to Input in multiple species. Motif with highest correlation to IgG motifs shown for each dataset. 6mA DIP-seq motifs in invertebrates show low correlation with IgG DIP-seq motifs whereas vertebrates show high correlation. (d) Barplot showing percent of motifs highly similar $(r>0.75)$ to $\operatorname{IgG}$ motifs for multiple species. (e) Proportion of short tandem repeats (STRs) in the genome of model organisms. STRs, short tandem repeats (f) Percentage reads mapping to mouse- or bacterial genomes. Boxplots represent median and top/bottom quartiles.

Figure 3. Biological impact of IgG correction. (a) Estimated false positive rate of enriched regions using $\mathrm{IgG}$ or Input as control. Bars represent mean \pm s.d of 2-7 biological replicates. (b) Overlap of $5 \mathrm{hmC}$ and $5 \mathrm{mC}$ regions using $\mathrm{IgG}$ or Input controls showing decreased overlap 
Lentini et al.

when using IgG controls. Venn diagram of $5 \mathrm{mC}$ and $5 \mathrm{hmC}$ overlap using $\mathrm{IgG}$ or Input controls (top). Below is a paired line plot of $5 \mathrm{mC}$ and $5 \mathrm{hmC}$ overlap using $\mathrm{IgG}$ or Input controls for multiple studies (indicated by symbols). Red markers indicate mean, $* \mathrm{P}<0.05$, paired T-test. $\boldsymbol{\Delta}=$ ERP000570, • = GSE31343, $\mathbf{a}=$ GSE24841, $\boldsymbol{\nabla}=$ GSE42250. (c) TET1 binding over IgG- or 5hmC regions using IgG or Input controls. (d) GO term enrichment of biological processes for $5 \mathrm{hmC}$ enriched regions in mESCs using either IgG or Input as controls showing stronger enrichment for developmental terms when using IgG controls and highly similar results to GO terms for $5 \mathrm{hmC}-\mathrm{Seal}$ and anti-CMS enriched regions. (e) Enrichment of ENCODE mESC histone ChIP-seq data relative to controls in regions enriched for IgG in DIPseq or random regions of same size and chromosome shows no difference in ChIP enrichment. Bars represent mean \pm s.d. of 26 biological replicates, P-values calculated using T-test. (f) Enrichment of ENCODE mESC histone ChIP-seq data for 5hmC- (left) or 5mC (right) enriched regions using $\mathrm{IgG}$ or Input as controls showing stronger association when using $\operatorname{IgG}$ controls. Data presented as mean (IgG) and bootstrap mean (Input) of 26 biological replicates, $* * * \mathrm{P}<1 \mathrm{e}-$ 5 , bootstrap resampling.

Figure 4. Antibodies in DIP-seq experiments bind repetitive elements which are incorrectly identified as enriched regions when not controlled for IgG binding. 
Lentini et al.

\section{SUPPLEMETARY FIGURE LEGENDS}

\section{Figure S1.}

(a, b) Immuno dot blot (a) and ELISA (b) of 5mC, 5hmC, $5 \mathrm{fC}$ and $5 \mathrm{caC}$ antibodies showing specificity for their respective mark in synthetic 426 bp oligos containing the different marks. (c) Enrichment of IgG or Input reads over the intersection of $5 \operatorname{modC}(5 \mathrm{mC}+5 \mathrm{hmC}+5 \mathrm{fC}+5 \mathrm{caC})$ enriched regions or non-intersecting $(5 \mathrm{mC} / 5 \mathrm{hmC} / 5 \mathrm{fC} / 5 \mathrm{caC})$ regions. $\mathrm{P}$-values calculated using T-test. (d) Correlation matrix of number of enriched DIP-seq regions per Mbp of mm9. All 5modC marks show correlation with IgG DIP-seq compared to Input. Correlation was calculated as Pearson correlation. (e) Venn diagram of overlapping enriched regions for $5 \mathrm{hmC}$, $5 \mathrm{mC}$ and $\mathrm{IgG}$ (left). Dinucleotide frequencies for overlapping regions, boxplots represent median and top/bottom quartiles (right). (f) Number of methylated $\mathrm{CpH}$ from WGBS data per enriched $\mathrm{IgG}$ or $5 \mathrm{mC}$ region. Boxplots represent median and top/bottom quartiles, P-values calculated using Mann-Whitney U-test. (g) Enrichment profile of IgG and 5hmC in TKO and WT mESCs over regions enriched for IgG showing highly similar profiles.

\section{Figure S2.}

(a) Estimated false positive rate for individual mESC or MEF datasets. *Estimated based on controls from mESCs. (b) False positive rate (FPR) was estimated based on the inverse fraction of regions identified by both Input and IgG versus total regions. (c) Venn diagram of enriched $5 \mathrm{hmC}$ regions in mESCs with different techniques showing higher overlap for 5hmC DIP-seq when using IgG controls. (d, e) Fraction of enriched 5modC regions identified using IgG or Input overlapping repetitive elements $(\mathbf{d})$ and dinucleotide repeats (e). Presented as mean \pm s.d of 2-7 biological replicates. ${ }^{*} \mathrm{P}<0.05, * * \mathrm{P}<0.01, * * * \mathrm{P}<0.001$, T-test. (f) GO term enrichment of biological processes for $5 \mathrm{hmC}$ enriched regions in mouse embryonic fibroblasts (MEFs) 
bioRxiv preprint doi: https://doi.org/10.1101/224279; this version posted November 24, 2017. The copyright holder for this preprint (which was not certified by peer review) is the author/funder. All rights reserved. No reuse allowed without permission.

\section{Lentini et al.}

using either IgG or Input controls. Stronger enrichment for developmental terms is observed when using the $\mathrm{IgG}$ controls. 


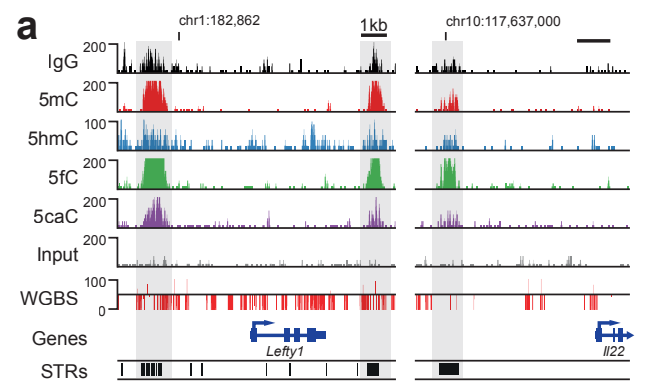

\section{Lentini et al. Figure 1}
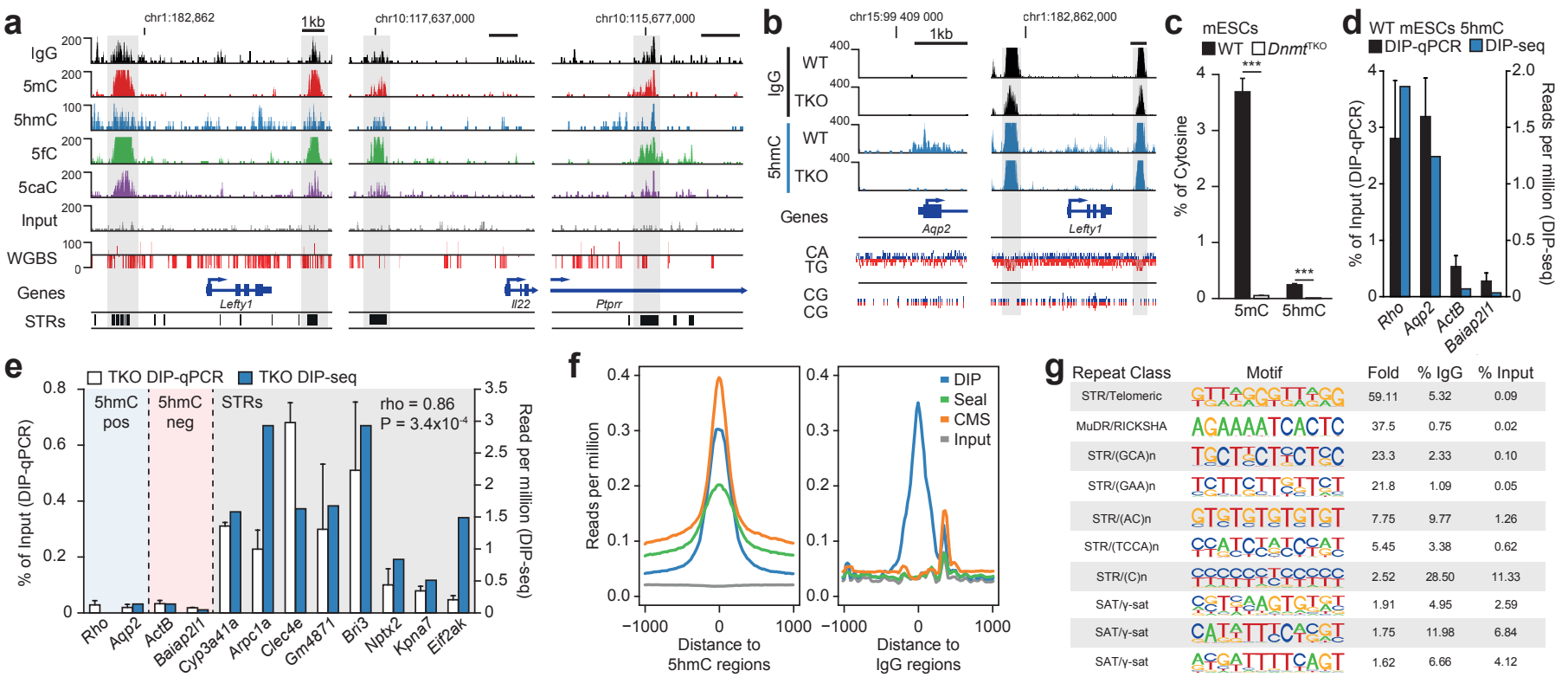

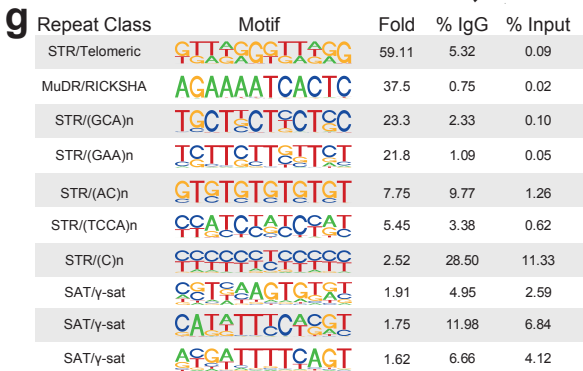




\section{Lentini et al. Figure 2}

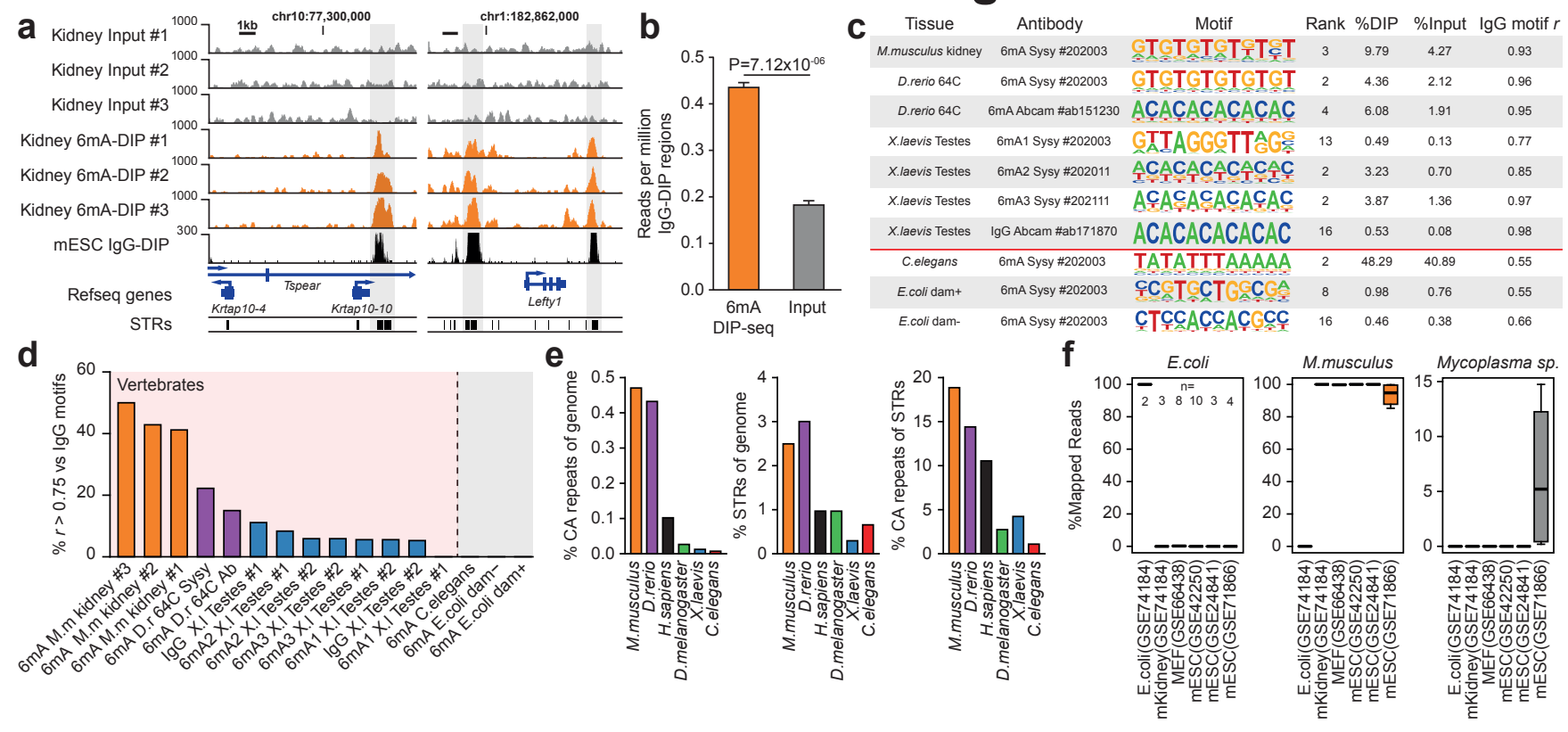




\section{Lentini et al. Figure 3}
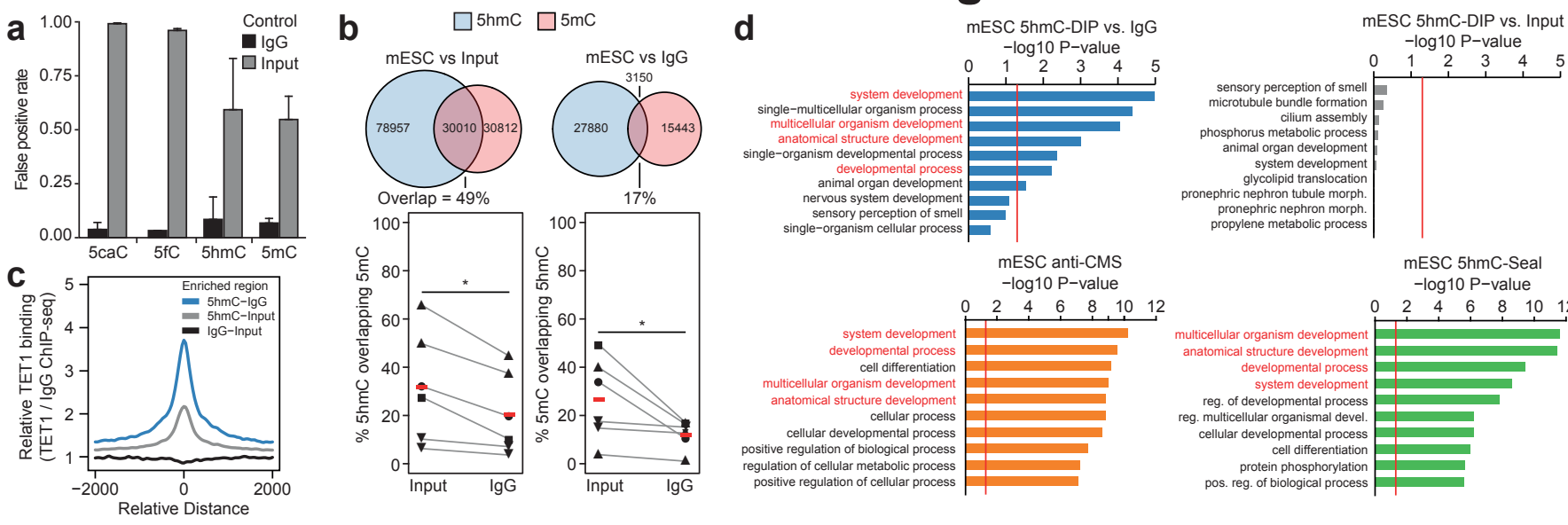

mESC 5 hmC-DIP vs. Input
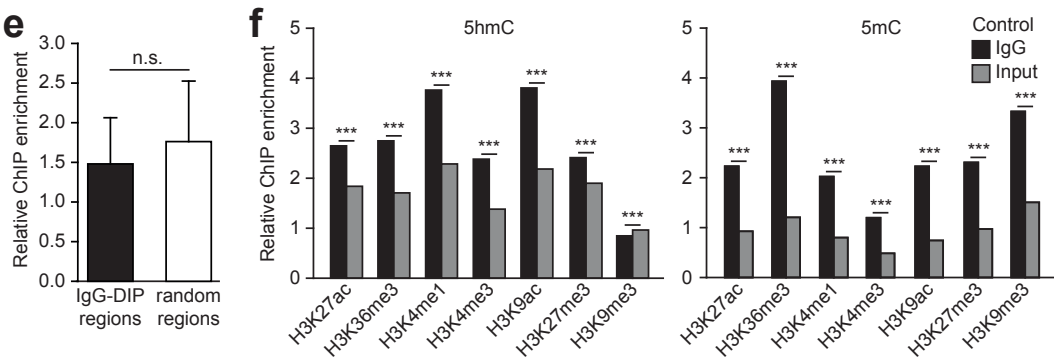

mESC anti-CMS

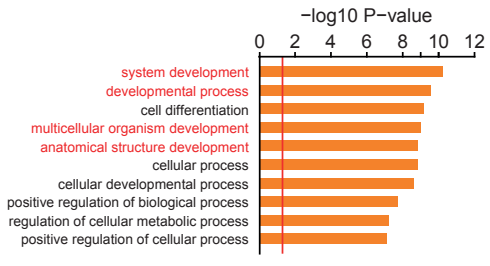

mESC 5hmC-Seal

$-\log 10 \mathrm{P}$-value

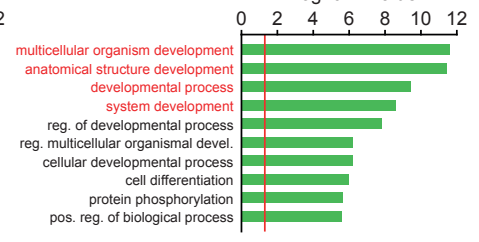


Lentini et al. Figure 4

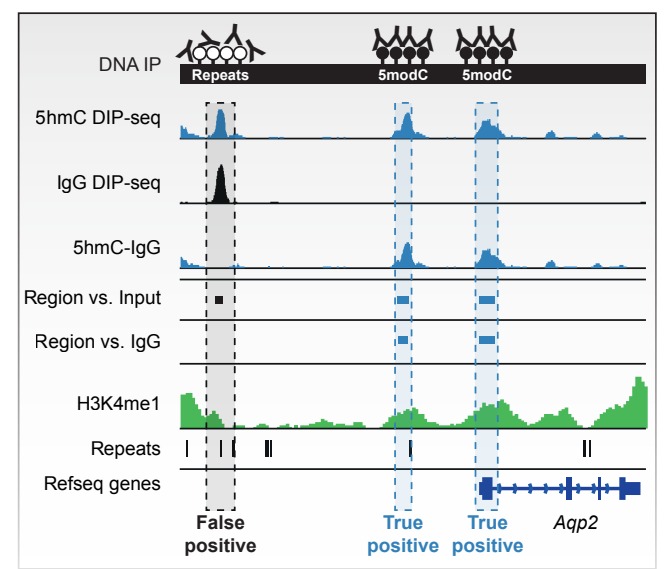


a

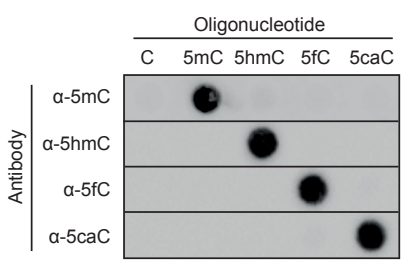

d

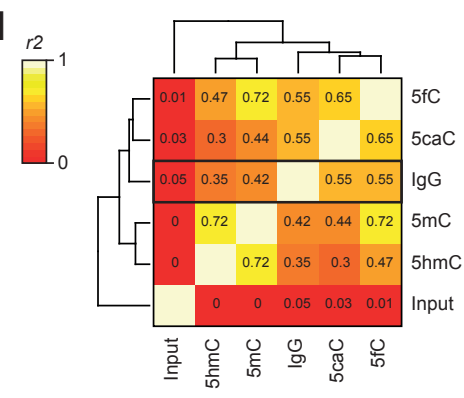

Lentini et al. Supplementary Figure 1

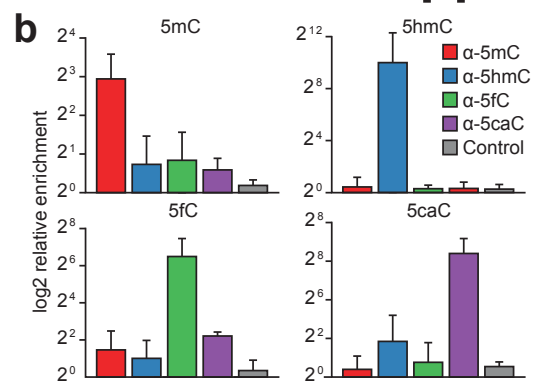

e

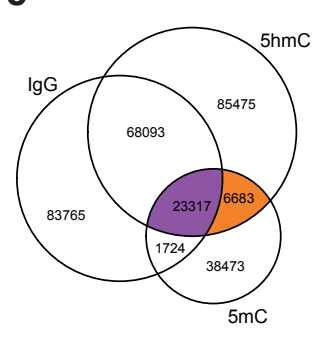

C

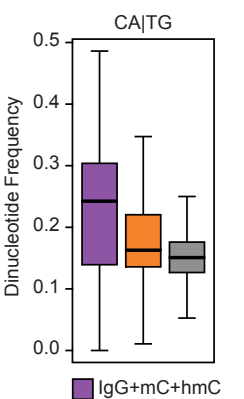

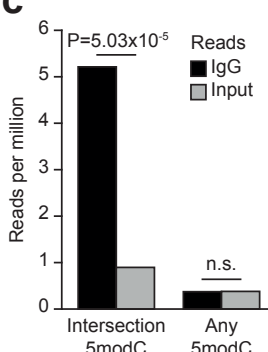

modc

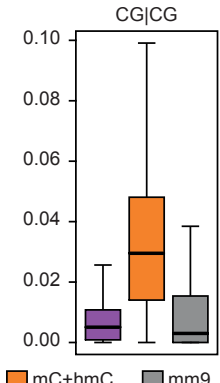

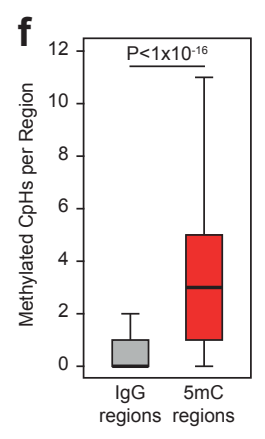

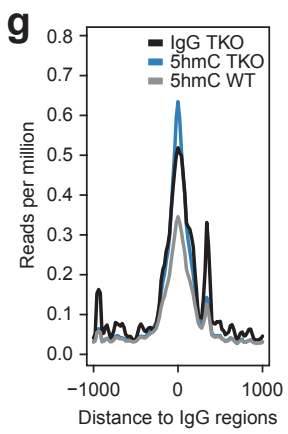


a

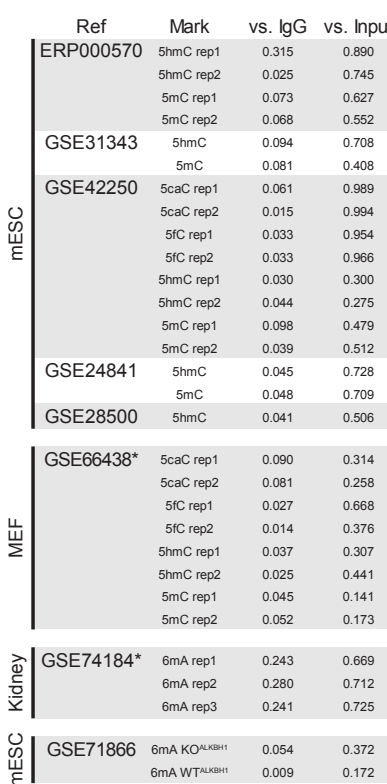

Lentini et al. Supplementary Figure 2 b

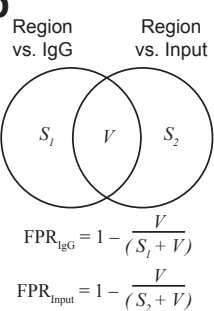

C
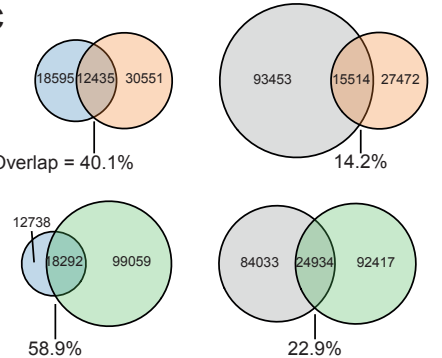

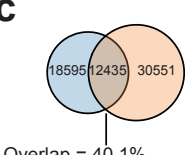

Enriched regions $\square$ DIP vs. IgG $\square$ DIP vs. Input $\square$ CMS $\square$ Seal
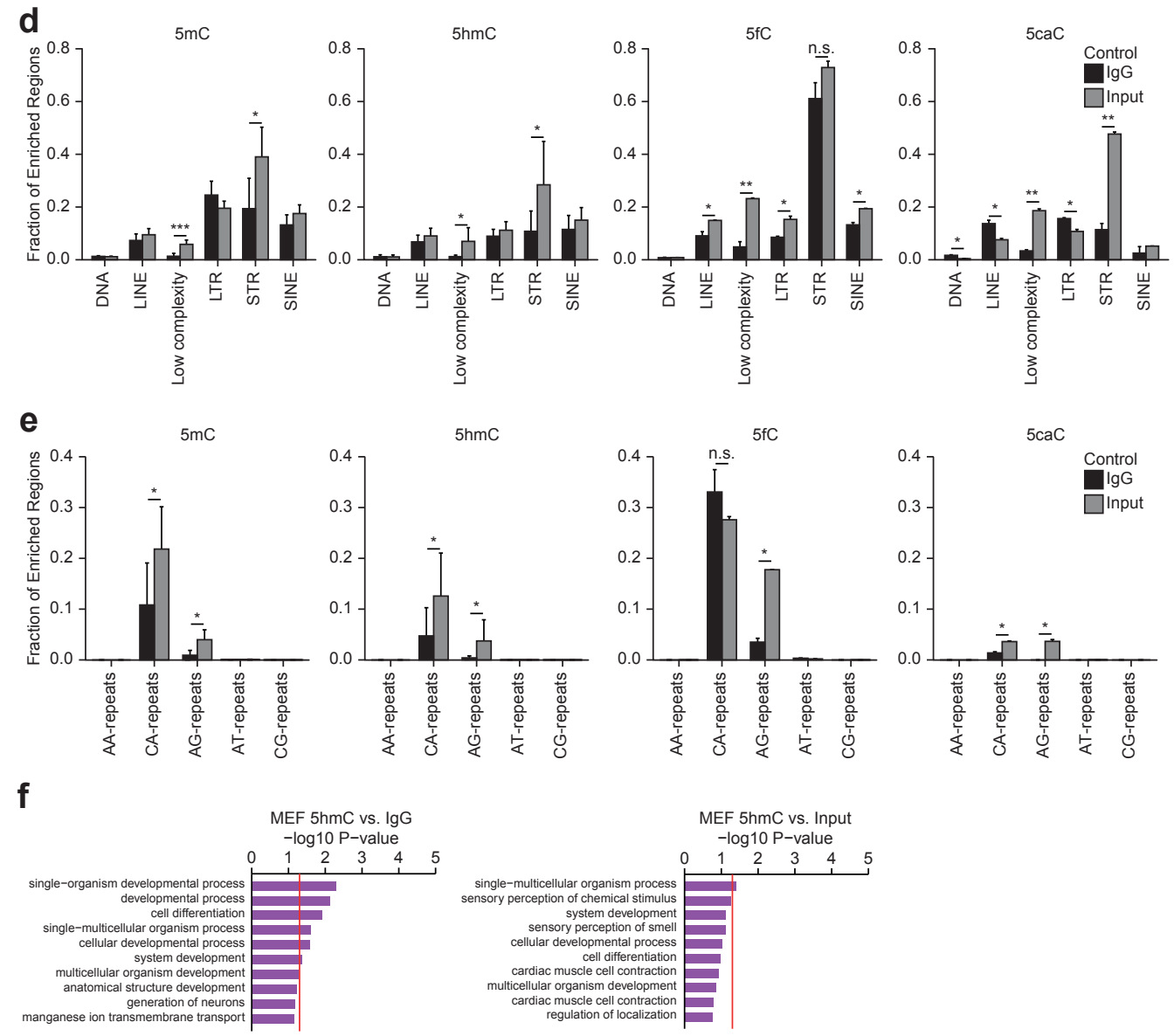


\section{SUPPLEMENTARY METHODS}

Cell culture. J1 mouse embryonic stem cells (mESCs; WT, male) were originally derived from the 129S4/SvJae strain. TKO (Dnmt1-/-, Dnmt3a-/-, Dnmt3b-/-) mESCs were derived from J1 mESCs ${ }^{46}$. Both cell lines were cultured in a humidified incubator at $5 \% \mathrm{CO}_{2}, 37^{\circ} \mathrm{C}$ on $0.2 \%$ gelatin coated tissue culture plastic in DMEM (Dulbecco's modified eagle medium) supplemented with $15 \%$ fetal calf serum, $0.1 \mathrm{mM}$ non-essential amino acids (Sigma-Aldrich, MI, USA), 1 mM sodium Pyruvate (Sigma-Aldrich, MI, USA), $1 \%$ Penicillin/Streptomycin, $2 \mathrm{mM}$ L-glutamine, $0.1 \mathrm{mM}$ beta-mercaptoethanol (Thermo Fisher, CA, USA), and ESGRO LIF (Millipore, MA, USA) at 500U/mL. mESCs were passaged every 2-3 days using trypsin/EDTA.

DNA extraction. Snap frozen cell pellets were treated with RNAse cocktail (Ambion, CA, USA) for 1 hour at $37^{\circ} \mathrm{C}$ followed by proteinase $\mathrm{K}$ treatment overnight at $55^{\circ} \mathrm{C}$. DNA was extracted by standard phenol chloroform/ethanol precipitation and eluted in TE.

DIP-qPCR. $1.5 \mu \mathrm{g}$ genomic DNA was sonicated to fragments ranging between 100-1000 bp with a peak at $400 \mathrm{bp}$ using a BioRuptor (Diagenode, Belgium), denatured at $95^{\circ} \mathrm{C}$ for $10 \mathrm{~min}$ then cooled on wet ice for $10 \mathrm{~min} .10 \%$ of samples were saved as Input and the remaining DNA was resuspended in 10x IP buffer (10 mM Na-Phosphate (mono-dibasic), $1 \% \mathrm{NaCl}, 0.05 \%$ Triton X-100, $\mathrm{pH}$ 7.0). Immunoprecipitations were performed using $1 \mu \mathrm{g}$ anti-5hmC antibody (Active Motif, \#39769) for $12 \mathrm{~h}$ at $4^{\circ} \mathrm{C}$ using constant rotation. Protein $\mathrm{G}$ dynabeads (Invitrogen, CA, USA, \#100-03D) were washed twice in 0.1\% PBS-BSA then added to the IP mixture for $1 \mathrm{~h}$ at $4^{\circ}$ using constant rotation. Beads were washed three times for $10 \mathrm{~min}$ using cold 1x IP buffer then resuspended in digestion buffer and incubated with $8 \mathrm{U}$ Proteinase K (New England Biolabs, MA, USA) for $2 \mathrm{~h} 1.5 \mathrm{~h}$ at $50^{\circ} \mathrm{C}, 800 \mathrm{rpm}$ in $50 \mathrm{mM}$ Tris, $10 \mathrm{mM}$ EDTA 0.5\% SDS, pH 8.0 and purified using DNA Clean \& Concentrator ${ }^{\mathrm{TM}}-5$ kit (Zymo Research, 
USA). Quantitative PCR was performed on a 7900HT real-time cycler (Applied Biosystems, CA, USA) using SYBR green master mix (Applied Biosystems, CA, USA). qPCR primers use Supplementary Table 4. hMeDIP qPCR primer sequences are listed in Supplementary Table 4, below.

\begin{tabular}{|l|l|l|l|}
\hline name & forward primer (5' - 3') & reverse primer (5' - 3') & designation \\
\hline Rho & ACCGTACAGCACAAGAAGCTGC & GAAGACCATGAAGAGGTCAGCC & True Positive \\
\hline Aqp2 & ATGTGGGAACTCCGGTCCATAG & GCCAAAGAAGACGAAAAGGAGC & True Positive \\
\hline Act $B$ & ATGAAGAGTTTTGGCGATGG & GATGCTGACCCTCATCCACT & True Negative \\
\hline Baiap $2 l 1$ & ATCTGCACTTGATGACAACTGG & CTTGTGAGACCAAGCTCTTAGC & True Negative \\
\hline Cyp3a4la & TTCACCTTTATGACTTGGTAGGC & GCTTCTCTTGTGAGGACTGTGG & False Positive \\
\hline Arpcla & TGGGGCTCATTTCTGTAATACC & TTCCATCTTCTCAAATCATTGC & False Positive \\
\hline Nptx2 & TCTCAAGTGCTGGGATTAAAGG & TCTGGGAAGCAAATCTAAGTCC & False Positive \\
\hline Gm4871 & CTGGTGTGTGTTTATCCTCAGC & AACTGTGGAGTGAGGTATGAAGG & False Positive \\
\hline Bri3 & TGGAGAGTGTGTATGTGTGAGC & AGGAGGCAGAAGGAGAAAGG & False Positive \\
\hline Clec4e & CACATACTGCCTTCTGCTATGC & TGTGTGAGTGAAAGGAGAGAGC & False Positive \\
\hline Kpna7 & CAACCAGGACTACACAGTGACG & GACACAGAAGCACAGAGAGAGG & False Positive \\
\hline Eif2ak & AGAGGCCAGAAGGTGTTGG & TTTCAGAGGACCTGAGTTTGG & False Positive \\
\hline
\end{tabular}

Quantification of cytosine modifications using mass spectrometry. $1 \mu \mathrm{g}$ of DNA was heat denatured at $100{ }^{\circ} \mathrm{C}$ for $5 \mathrm{~min}$ in $20 \mu \mathrm{L} \mathrm{H}_{2} \mathrm{O}$ then immediately cooled on ice. $10 \mu 1 \mathrm{P} 1$ Nuclease (0.02 $\mathrm{U} / \mu 1$ in $\left.90 \mathrm{mM} \mathrm{AmAc,} 0.3 \mathrm{mM} \mathrm{ZnSO}_{4}, \mathrm{pH} 5.3\right)$ was added followed by incubation at 50 ${ }^{\circ} \mathrm{C}$ for $2 \mathrm{~h} .10 \mu \mathrm{l}$ Alkaline phosphatase $(0.08 \mathrm{U} / \mu \mathrm{l}$ in $200 \mathrm{mM}$ TRIS-HCl, $0.40 \mathrm{mM}$ EDTA, pH 8) was added followed by incubation at $37^{\circ} \mathrm{C}$ for $30 \mathrm{~min}$. Proteins were precipitated by the addition of $160 \mu \mathrm{l}$ cold acetonitrile. Following centrifugation at $17000 \mathrm{x}$ g for $5 \mathrm{~min}, 180 \mu \mathrm{l}$ of the supernatant was evaporated under nitrogen and reconstituted in $40 \mu 10.1 \%$ formic acid. The chromatographic system consisted of an Acquity UPLC (Waters, MA, USA) and a Xevo triple quadrupole mass spectrometer (Waters, MA, USA). The extracts were separated on an HSS T3 column (150x2.1 mm, $1.7 \mu \mathrm{m}$, Waters, MA, USA) at $45^{\circ} \mathrm{C}$ and a flow rate of 450 
$\mu 1 /$ min using a gradient elution with $0.05 \%$ acetic acid and methanol, $0-1.3 \min 2 \% \mathrm{~B} ; 1.3-5.5$ $\min 2-9 \% \mathrm{~B} ; 5.5-7.5 \mathrm{~min}$ re-equilibration at $2 \% \mathrm{~B}$. For $\mathrm{dC}$ a $1 \mu \mathrm{l}$ injection was made and for $\mathrm{mC}, \mathrm{hmC}, \mathrm{fC}$ and $\mathrm{caC}$ a $15 \mu \mathrm{l}$ injection was made. Analytes were detected in the multi reaction monitoring (MRM) mode using three time windows with the following transistions 0-2.3 min - C (228->95 \& 228->112) and hmC (258->124 \& 258->142); 2.3-4 min - mC (242->109, 242->126) and $\mathrm{caC}(272->138,272->156) ; 4-7.5 \mathrm{~min}$ - fC (256->97, 256->140).

Immuno dot-blot. $10 \mathrm{ng} 426$ bp oligos containing $5 \mathrm{mC}, 5 \mathrm{hmC}, 5 \mathrm{fC}, 5 \mathrm{caC}$ or $\mathrm{C}$ (GeneTex, $\mathrm{CA}$, USA) was denatured at $95^{\circ} \mathrm{C}$ for $15 \mathrm{~min}$ in $0.4 \mathrm{M} \mathrm{NaOH}$ and $10 \mathrm{mM}$ EDTA then immediately cooled on ice. Samples were applied to a positively charged nylon membrane under vacuum using a Dot Blot Hybridisation Manifold (Harvard Apparatus, MA, USA). The membranes were briefly washed in $2 \mathrm{X}$ SSC buffer $(0.3 \mathrm{M} \mathrm{NaCl}, 30 \mathrm{mM}$ NaCitrate $)$ then crosslinked using a UV Stratalinker 1800 (Stratagene, CA, USA) and baked at $80^{\circ} \mathrm{C}$ for 2 hours. Membranes were blocked in casein blocking buffer (Li-Cor) for $15 \mathrm{~min}$ at $4^{\circ} \mathrm{C}$ then incubated with an antibody against 5mC (1:3000, Zymo \#A3001), 5hmC (1:3000, ActiveMotif \#39791), 5fC (1:3000, ActiveMotif \#61227) or 5caC (1:3000, ActiveMotif \#61229) for $1 \mathrm{~h}$ at $4{ }^{\circ} \mathrm{C}$. Membranes were washed 3 times for 5 min in TBS-Tween $(0.05 \%)$ then incubated with a HRP conjugated goat-anti-rabbit antibody for 5hmC, $5 f C$ and 5caC (1:3000, Bio-Rad \#1706515) or goat-anti-mouse for 5mC (1:3000, Bio-Rad \#1706516). Following treatment with Clarity Western ECL substrate (Bio-rad, CA, USA), membranes were scanned on a ChemiDoc MP imaging system (Bio-Rad, CA, USA).

ELISA. 426 bp dsDNA oligos containing 5mC, 5hmC, 5fC, 5cacC or C (GeneTex, CA, USA) was diluted to a concentration of $50 \mathrm{ng} / \mathrm{mL}$ in coating buffer ( $1 \mathrm{M} \mathrm{NaCI}, 50 \mathrm{Mm} \mathrm{Na}_{2} \mathrm{PO}_{4}, 0.02 \%$ (w/v) $\mathrm{NaN}_{3}, \mathrm{pH} 7.0$ ) then $50 \mu 1$ were placed into each well of black 96-well plates (4titude, UK) and incubated overnight at $37^{\circ} \mathrm{C}$. Plates were blocked for $1 \mathrm{~h}$ at room temperature in Blocker Casein in PBS (Thermofischer Scientific, MA, US) followed by washing with $100 \mu$ PBS 
containing $0.1 \%(\mathrm{v} / \mathrm{v})$ Tween 20 . Wells were incubated with $50 \mu \mathrm{l}$ of their respective antibodies (1:1000, see above) for $1 \mathrm{~h}$ at room temperature, then washed 3 times and incubated with $50 \mu 1$ of horseradish peroxidase (HRP)-conjugated goat-anti- mouse or goat-anti- rabbit antibody (1:5000, see above) for $30 \mathrm{~min}$. Plates were treated with $70 \mu 1$ of Clarity Western ECL substrate (Bio-rad, CA, USA) for 5 min then scanned in a Spark 10M multimode microplate reader (Tecan Trading AG, Switzerland).

Uniform analysis pipeline for processing of published DIP-Seq data. Raw 5 modC DIP-seq sequencing data was downloaded from GSE42250, GSE24841, GSE31343, ERP000570, GSE28500 and aligned to the mouse genome (mm9) using Bowtie $2^{47}$ (bowtie2 -N 1 -L 30). Genomic coverage was calculated using Bedtools ${ }^{48}$ (bedtools genomecov -bg -split) then normalized as reads per million mapped (RPM) for visualization. Identification of enriched regions was performed using MACS2 $2^{49}$ (macs2 --bw=200 -p 1e-5) using IgG or Input controls from the same study where possible otherwise IgG or Input samples from the above studies were pooled and randomly subsampled to 20 million reads as controls. Unless otherwise stated, 5modC enriched regions were identified using $\operatorname{IgG}$ controls and $\mathrm{IgG}$ enriched regions using Input.

6mA DIP-seq data was downloaded from GSE71866, GSE74184 and GSE76740 and processed as 5modC DIP-seq data (see above).

Bisulfite sequencing data was obtained from GSE41923 and aligned to a bisulfite converted mm9 index using Bismark ${ }^{50}$ (bismark $-\mathrm{N}$ 1). Methylation levels of Cytosines in both $\mathrm{CpG}$ and non-CpG contexts were extracted (bismark_methylation_extractor) and bases with at least $5 \mathrm{X}$ coverage were used for analysis. 
Raw 5hmC-Seal data was downloaded from GSE41545 and processed as DIP-seq data (see above) and anti-CMS was downloaded from GSE28682 and aligned using Bismark ${ }^{50}$ with the same settings as for DIP-seq (bismark -N 1 -L 30).

TET1 ChIP-seq data was downloaded from GSE24843 and histone ChIP-seq data for mESCs was obtained from the ENCODE project ${ }^{51}$ and processed as DIP-seq data (see above).

See Supplementary Table 1 for specification of files used for each analysis/figure.

Estimation of falsely enriched regions. Enriched regions were obtained from MACS2 using either pooled IgG or Input from mESCs as control (see above). True regions were considered when an overlapping region was called for both $\operatorname{IgG}$ and Input controls and falsely enriched regions were calculated as the inverse fraction for either control (Supplementary Fig. 2b).

Motif enrichment of FASTQ files. FASTQ files were trimmed of adapters using ea-utils ${ }^{52}$ (fastq-mcf -x 0 -q 0 -k 0 -s 4.6) then randomly subsampled to 1 million reads and subjected to de novo motif enrichment analysis using Homer $2^{53}$ (homer2 denovo -len 12). Input samples from the same study was used as background when available, otherwise a pooled input from multiple studies was used (see above). Correlation between motif PWMs was performed using Pearson correlation as implemented in TFBStools ${ }^{54}$ (PWMsimilarity), subject motifs were repeated once to account for base shifts. To identify if identified motifs belong to a certain repeat class, motif PWMs were mapped to repeats in mouse (RepBase v22.0155) using Homer $2^{53}$ (scanMotifGenomeWide.pl).

Taxonomic annotation of sequence reads. Taxonomic annotation of raw sequencing reads was performed by aligning reads to a custom reference genome of $\mathrm{mm} 9$ combined with the bacterial genomes of Mycoplasma species M.arginini (ASM154797v1), M.hyorhinis_dbs 1050 (ASM49681v1), M.hyorhinis_gdl1 (ASM24112v1), M.hyorhinis_hub1 (ASM14570v1), M.hyorhinis_mcld (ASM21129v1), M.hyorhinis_sk76 (ASM31363v1) as well as E.coli 
(HUSEC2011CHR1) using Bowtie $2^{47}$, with the same settings as outlined above. For determination of short tandem repeat (STR) fraction of species genomes, Tandem Repeat Finder $^{56}$ (TRF) results for genomes (ce10, danRer10, dm6, hg38, mm10) was obtained from UCSC. For Xenopus laevis, the genomic sequence was obtained from Xenbase (Xenla9.1) and STRs was identified using TRF 4.09 with recommended settings and a maximum period size of $12(\operatorname{trf} 27780105012)$.

GO term enrichment analysis. Top 500 enriched regions were mapped to the nearest gene within $10 \mathrm{~kb}$ and enrichment of GO terms biological processes was performed using PANTHER $^{57}$ with default settings.

Statistical analysis. All statistical analysis was performed using the statistical programming language $\mathrm{R}^{58}$ unless otherwise stated. $\mathrm{P}$-values $<0.05$ were considered significant.

\section{References}

46. Tsumura, A. et al. Maintenance of self-renewal ability of mouse embryonic stem cells in the absence of DNA methyltransferases Dnmt1, Dnmt3a and Dnmt3b. Genes to cells : devoted to molecular \& cellular mechanisms 11, 805-814 (2006).

47. Langmead, B. \& Salzberg, S.L. Fast gapped-read alignment with Bowtie 2. Nature methods 9, 357-359 (2012).

48. Quinlan, A.R. \& Hall, I.M. BEDTools: a flexible suite of utilities for comparing genomic features. Bioinformatics 26, 841-842 (2010).

49. Zhang, Y. et al. Model-based analysis of ChIP-Seq (MACS). Genome biology 9, R137 (2008).

50. Krueger, F. \& Andrews, S.R. Bismark: a flexible aligner and methylation caller for Bisulfite-Seq applications. Bioinformatics 27, 1571-1572 (2011).

51. Yue, F. et al. A comparative encyclopedia of DNA elements in the mouse genome. Nature 515, 355-364 (2014).

52. Aronesty, E. Comparison of Sequencing Utility Programs. The Open Bioinformatics Journal 7, 1-8 (2013).

53. Heinz, S. et al. Simple combinations of lineage-determining transcription factors prime cis-regulatory elements required for macrophage and B cell identities. Molecular cell 38, 576-589 (2010).

54. Tan, G. \& Lenhard, B. TFBSTools: an R/bioconductor package for transcription factor binding site analysis. Bioinformatics 32, 1555-1556 (2016).

55. Bao, W., Kojima, K.K. \& Kohany, O. Repbase Update, a database of repetitive elements in eukaryotic genomes. Mobile DNA 6, 11 (2015).

56. Benson, G. Tandem repeats finder: a program to analyze DNA sequences. Nucleic acids research 27, 573-580 (1999). 
bioRxiv preprint doi: https://doi.org/10.1101/224279; this version posted November 24, 2017. The copyright holder for this preprint (which was not certified by peer review) is the author/funder. All rights reserved. No reuse allowed without permission.

Lentini et al

Supplementary Methods

57. Mi, H., Muruganujan, A. \& Thomas, P.D. PANTHER in 2013: modeling the evolution of gene function, and other gene attributes, in the context of phylogenetic trees. Nucleic acids research 41, D377-386 (2013).

58. R Development Core Team R: A language and environment for statistical computing. R Foundation for Statistical Computing, Vienna, Austria. ISBN 3-900051-07-0. http://www.R-project.org (2008). 